\title{
Uncommon branching pattern of the hepatic arteries in a living donor: a case report and brief literature review
}

\author{
M. Morawski1, 2, R. Stankiewicz ${ }^{1}$, B. Ciszek ${ }^{2}$, R. Pacho³ ${ }^{3}$ W. Patkowski ${ }^{1}$, M. Krawczyk ${ }^{1}$ \\ ${ }^{1}$ Chair and Department of General, Transplant and Liver Surgery, Medical University of Warsaw, Poland \\ ${ }^{2}$ Department of Descriptive and Clinical Anatomy, Centre of Biostructure Research, Medical University of Warsaw, Poland \\ ${ }^{3}$ Second Department of Clinical Radiology, Medical University of Warsaw, Poland
}

[Received: 27 January 2015; Accepted: 2 March 2015]

\begin{abstract}
Numerous variations of the hepatic arteries are common in surgical patients. We present a 35-year-old woman who was admitted to our department in order to assess possibility of becoming living donor. Preoperative computed tomography scan revealed anomalous branching pattern of the hepatic arteries. In this case right posterior sectoral artery has been given off by the greater pancreatic artery, left hepatic artery has been replaced by the artery arising from the left gastric artery and double segment 4 branches have been observed. To the best of our knowledge, this pattern has not been described in the literature, yet. (Folia Morphol 2016; 75, 1: 125-129)
\end{abstract}

Key words: hepatic artery, variations, living donor liver transplantation, multidetector computed tomography angiography

\section{INTRODUCTION}

The blood supply to the liver is dependent on two sources: approximately $1 / 4$ comes from hepatic arteries whereas $3 / 4$ is delivered by portal vein. These vessels conduct high-oxygenated, high-pressure and low-oxygenated, low-pressure blood, respectively. On the basis of the branching pattern of the vessels and biliary ducts that enter and leave hepatic parenchyma, liver has been divided into 4 sectors and 8 segments by Couinaud $[2,5]$. Although numerous divisions have been proposed before and after Couinaud [5], the bulk of them have no clinical significance and remain the source of terminological confusion. Detailed knowledge of normal and uncommon branching pattern of hepatic vessels and biliary ducts is essential for safe hepatic resection and helpful in preoperative assessment of patients that are at risk of accidental injury of aberrant arteries or veins. Surgeons have developed numerous techniques, including the Carrel patch, to retain during liver harvesting accessory or replaced arteries stemming from the superior mesenteric artery (SMA) or left gastric artery (LGA) and passing through the head of the pancreas and pars densa of the lesser omentum, respectively [7].

The biggest visceral branch of the abdominal aorta, coeliac trunk (CTr), is the main source of arterial blood reaching the liver. Most commonly, it ramifies into LGA, splenic artery (SA) and common hepatic artery (CHA). The latter gives rise to proper hepatic artery that eventually branches into left and right hepatic arteries entering liver parenchyma. According to Michels [14], aforementioned 'textbook description' occurs approximately in one half (55\%) of population, whereas in the remaining numerous variations are encountered. The latter group comprises individuals in whom accessory or replaced hepatic arteries are present. Regardless of smaller diameter of accessory arteries in comparison 
to right and left hepatic arteries, they have own area of distribution and their disruption may lead to severe complications [6, 13].

Because of shortage of cadaveric livers, living donor liver transplantation (LDLT) or split liver transplantation (SLT) are widely accepted ways of treatment of end-stage liver disease. LDLT is performed most frequently by harvesting segments 2 and 3 (left hepatectomy) in children, whereas in adult numerous different techniques have been described [19]. During hepatectomy variants of hepatic arteries should be kept in mind in order to provide both the graft and the remnant with undamaged vascularisation [17].

Preoperative radiologic assessment of the branching pattern of hepatic arteries is crucial for evaluation of the presence of uncommon variations in origin and distribution of arterial branches and helps surgeon to establish patients to be excluded from donor pool because of disadvantageous hepatic artery, portal vein or biliary branching pattern, although this possibility is relatively rare. In presented case we discuss a living donor in whom radiological imaging revealed atypical branching pattern of hepatic arteries.

\section{CASE REPORT}

A 33-year-old woman was admitted to the Department of General, Transplant and Liver Surgery in Warsaw in order to assess possibility of becoming living donor and feasibility of safe left hepatectomy. Procedure of donor evaluation comprised computed tomography (CT) imaging of abdominal cavity which is the source of information concerning liver's mass, volume and vascular anatomy. Determination of the size allows surgeon to predict whether harvested segments' volume is sufficient to meet recipient's needs, whereas knowledge of vascular anatomy helps to assess feasibility of reanastomosis in recipient. Investigation have been performed using GE Lightspeed PRO 16 CT Scanner.

In our patient CT angiography has been performed and revealed uncommon branching pattern of arteries entering liver parenchyma. The division of the CTr was typical and its trifurcation into the LGA, SA and CHA could be observed (Figs. 1, 2). After short extraperitoneal running along the superior margin of the head of the pancreas, the CHA entered the space between two laminae of the hepatoduodenal ligament. Posterior to the pylorus, before entering the lesser omentum, the CHA gave rise to the gastroduodenal artery that ran inferiorly between the head of the pancreas and descending part

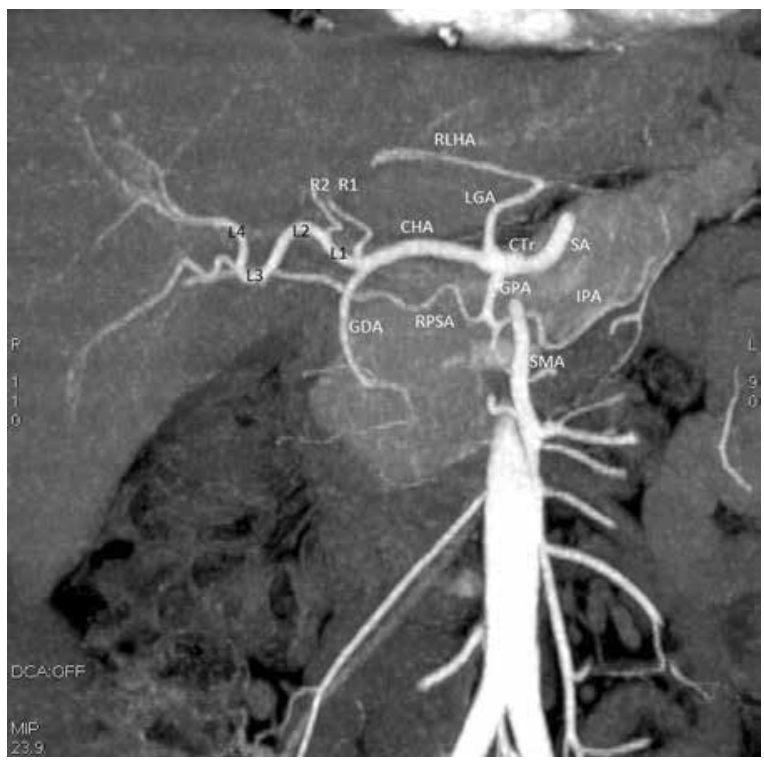

Figure 1. Reconstruction of the upper abdomen arterial vasculature. Coeliac trunk (CTr) giving off common hepatic artery (CHA), splenic artery (SA) and left gastric artery (LGA). Nearby its origin the SA gives off greater pancreatic artery (GPA) that divides into right posterior sectoral artery (RPSA) and inferior pancreatic artery (IPA), eventually. CHA branches into gastroduodenal artery (GDA) and a branch forming 4 loops (L1, L2, L3, L4) supplying right anterior sector. Two ramus to segment $4(R 1, R 2)$ are given off by $L 1$. There is also replaced left hepatic artery (RLHA) stemming from the LGA. Superior mesenteric artery (SMA) does not give any aberrant vessel to the liver.

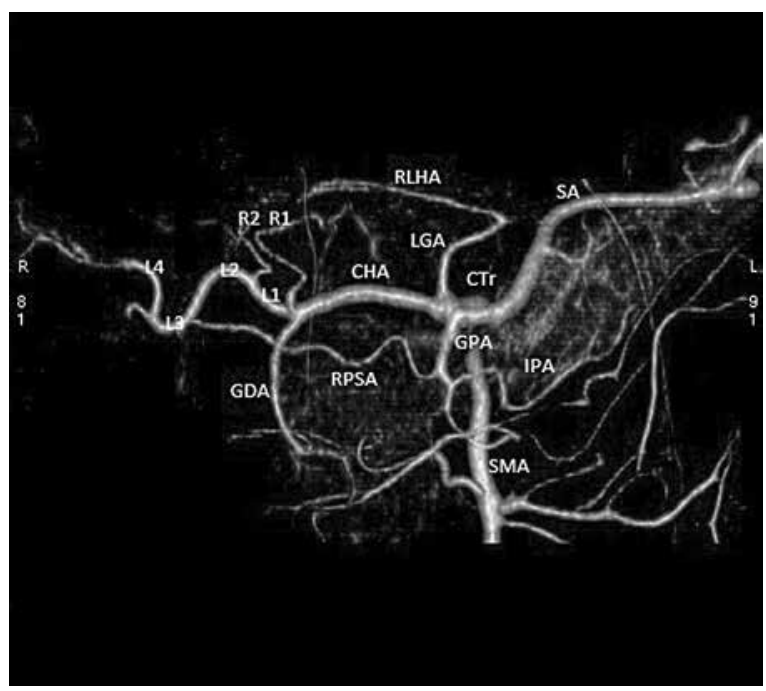

Figure 2. Reconstruction of the upper abdomen arterial vasculature; SMA - superior mesenteric artery; $\mathrm{CTr}$ - coeliac trunk; SA splenic artery; GPA — greater pancreatic artery; IPA — inferior pancreatic artery; LGA — left gastric artery; CHA — common hepatic artery; GDA — gastroduodenal artery; RPSA — right posterior sectoral artery, RLHA — replaced left hepatic artery; L1, L2, L3, L4 - loops of the branch supplying right anterior sector, $\mathrm{R} 1, \mathrm{R} 1$ - two ramus to segment 4 . 

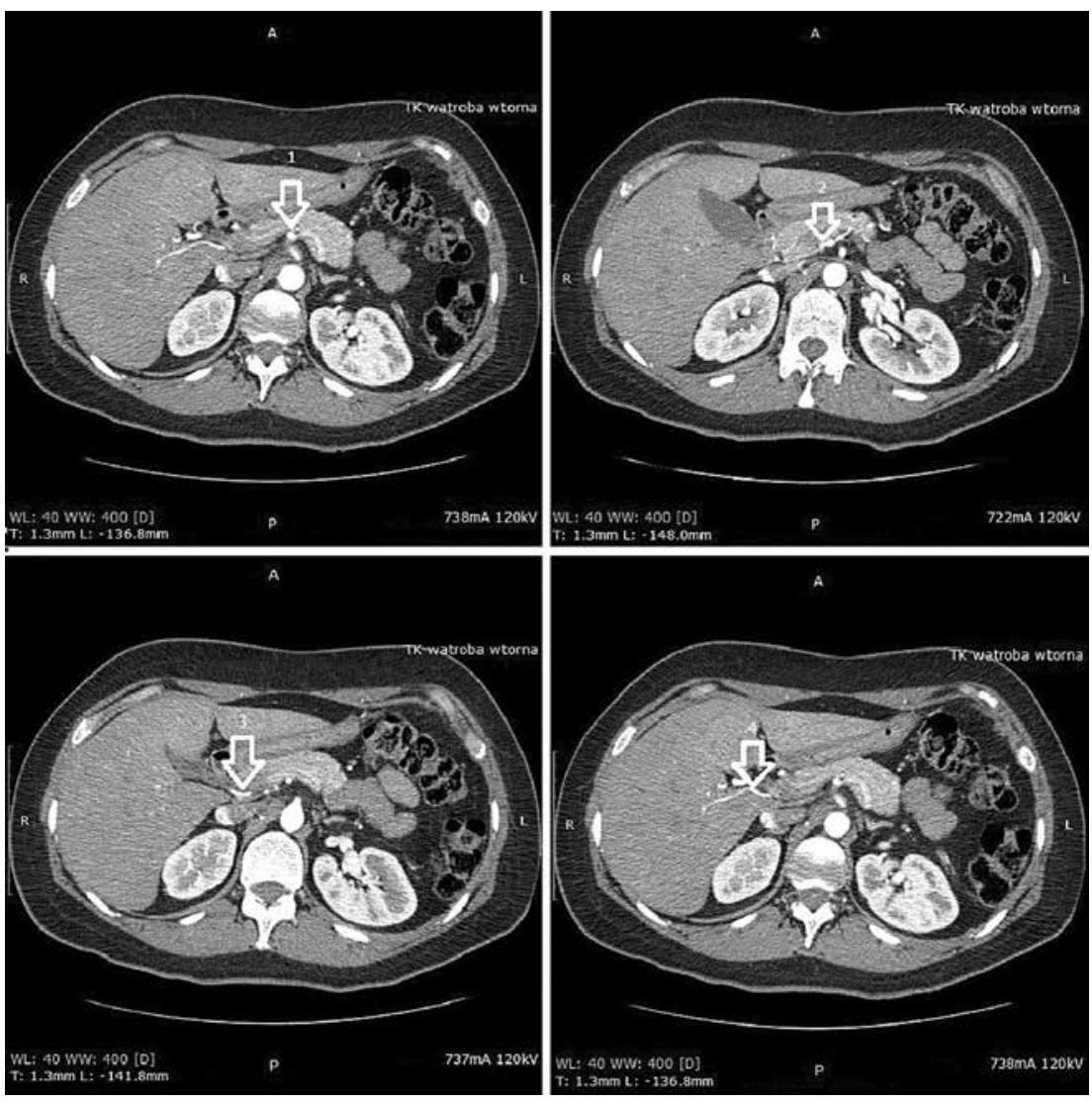

Figure 3. Cross sections of the upper abdomen showing the origin of the right posterior sectoral artery (RPSA) (2) from the greater pancreatic artery (1). RPSA runs posteriorly to the head of the pancreas (3) and the portal triad, eventually reaching liver parenchyma (4).

of the duodenum and divided into the superior anterior pancreaticoduodenal artery and the right gastroepiploic artery, eventually. The second branch of the CHA that ran in hepatoduodenal ligament toward liver hilum in this particular case could be divided into 4 sections called loops. The first loop was bordered proximally by its origin from the CHA and distally by a small artery running toward fissure of round ligament. The latter vessel was accompanied by similar parallel one that stemmed from concave surface of the first loop, nearby its origin from the $\mathrm{CHA}$. It also gave off right gastric artery. $\mathrm{CT}$ findings suggested these arteries supplied mainly segments 3 and $4 \mathrm{~b}$ of the liver. Similarly to the first 2 loops, the second and the third ones were separated by origin of the cystic artery. There is no evident border between the last 2 loops although it could be established arbitrarily in the middle of the distance separating the apices of the 3rd and the 4th loops. The 4th loop stated the last extrahepatic section of the artery reaching and supplying the area of the anterior sector of the liver (segment 5 and 8 according to Couinaud), eventually.
The second branch of the CTr - the LGA ran superiorly rising parietal peritoneum and forming gastropancreatic fold. At the level of gastroesophageal junction the artery turned 180 degrees and continued descending along the lesser curvature of the stomach. The most cephalad section of LGA - a so-called genu - gave off arterial branch running within 2 laminae of the pars densa of the lesser omentum. The artery reached the fissure of venous ligament and divided into 2 vessels providing with arterial blood mainly segments 2 and $4 a$, eventually.

In our patient the biggest branch of the $\mathrm{CTr}$ - the $\mathrm{SA}$ - also participated in providing the liver with arterial blood. Nearby its origin the SA gave off a though branch - the greater pancreatic artery (GPA). Behind the head of the pancreas the GPA gave rise to an artery that lied transversely and posteriorly to the portal vein, the body and the head of the pancreas. At last it ran within the lesser omentum and reached the porta hepatis and the liver itself where it divided into branches supplying right posterior sector, predominantly (Fig. 3). 


\section{DISCUSSION}

Development in liver surgery we witnessed thanks to improvement in liver transplantation and hepatic resection techniques forced anatomists to look again at vascular anatomy of the upper abdomen. Before the pattern of $\mathrm{CTr}$ branches is to be given consideration, we should define what "accessory" and "replaced" artery mean. According to Wang et al. [18], the term "accessory" is used only for an aberrant artery in case of its coexistence with typical hepatic branch from the CTr. Logically, if the right (RHA) or the left hepatic arteries (LHA) are absent the term "replaced" should be used.

The well-known, vast description and classification of the CTr's branches was carried out by Adachi [1]. On the basis of the origin of the 4 main arteries (Hauptarterien): the SMA, SA, LGA and CHA, 6 types can be distinguished. In our case typical trifurcation of the CTr into the SA, LGA and CHA and separate origin of SMA can be observed. This pattern should be classified as type 1 according to Adachi and named CTr or hepatogastrosplenic trunk. It is encountered in $55 \%$ to $77.5 \%[4,8-10]$ of cases. Because of numerous aberrant vessels arising from CHA, LGA and GPA it is not clear whether described pattern matches the criteria of membership in one of 11 groups in type 1. There is no problem with the artery leaving the genu of the LGA that should be named left replaced hepatic artery due to the lack of the typical LHA.

In addition, there are still unsolved questions about 2 branches arising from the first loop of aforementioned sinuous branch of the $\mathrm{CHA}$ and about the RHA itself. These 2 small branches supplying segments 3 and $4 \mathrm{~b}$ resemble double middle hepatic artery (MHA; ramus medius according to Adachi). Michels defined MHA as a hepatic branch that runs in the fissure of round ligament and supplies the quadrate lobe [4, 7]. Although, the literature reveals discrepancy in descriptions of anatomy of the MHA, numerous anatomical studies used the term MHA synonymously for segmental branch supplying segment 4 and claimed that this vessel originates from the LHA, predominantly $(54-61.5 \%)[10,15]$. However, according to Kamel et al. [11] arterial supply to segment 4 originates mainly from RHA (62.5\%) [10].

Michels [14] classification of hepatic arteries in Hiatt's modification marks out 6 types (Table 1). Although our patient had pattern that could be classified as type 4 , it does not meet the criteria due to the fact that the branch of the GPA, defined on the
Table 1. Hiatt's classification of hepatic arteries

\begin{tabular}{llc}
\hline Type & Description & Per cent \\
\hline 1 & 'Textbook type' & $75.7 \%$ \\
2 & Replaced/accessory LHA & $9.7 \%$ \\
3 & Replaced/accessory RHA & $10.6 \%$ \\
4 & Replaced/accessory LHA + & $2.3 \%$ \\
& + replaced/accessory RHA & \\
5 & CHA from SMA & $1.5 \%$ \\
6 & CHA from aorta & $0.2 \%$ \\
\hline
\end{tabular}

CHA — common hepatic artery; LHA — left hepatic artery; RHA — right hepatic artery; SMA — superior mesenteric artery

basis of supplied area as right posterior sectoral artery or right accessory hepatic artery, does not stem from SMA.

In addition, in this particular case the harvested graft, comprising segments 2 and 3 according to Couinaud, was supplied by 2 hepatic arteries: LRHA and one branch to segment 4 ( $2 \mathrm{~mm}$ and $2.5 \mathrm{~mm}$, respectively). Because sufficient anastomoses between these arteries were absent 2 end-to-end anastomoses with donor's vessels have been performed using Surgipro $8 / 0$. Hepatic function following arterial reperfusion was satisfactory.

\section{CONCLUSIONS}

This short discussion showed the pattern encountered in our patient does not fulfil the criteria of main existing classifications. In addition, this case presents the origin of right posterior sectoral artery that have not been described yet. Unclassified arterial variation state $1.5 \%$ to $4.6 \%[3,6,12,16]$. We hope our finding will enrich this group and enable thorough meta-analysis of arterial variability in this region and preparation of new classification integrating the rarest branching patterns.

\section{REFERENCES}

1. Adachi B (1928) Das Arteriensystem der Japaner. Verlag der kaiserlich-japanischen Universität zu Kyoto, Kyoto, pp. $18-46$.

2. Aleksandrowicz R (1964) Vascular segments of the liver. Folia Morphol, 23: 29-42.

3. Arjhansiri K, Charoenrat P, Kitsukjit W (2006) Anatomic variations of the hepatic arteries in 200 patients done by angiography. J Med Assoc Thai, 89 (suppl. 3): S161-S168.

4. Chen H, Yano R, Emura S, Shoumura S (2009) Anatomic variation of the celiac trunk with special reference to hepatic artery patterns. Ann Anat, 191: 399-407. doi: 10.1016/j.aanat.2009.05.002. 
5. Couinaud C (1954) Lobes et segments hepatiques. Press Med, 62: 709-712.

6. De Cecco CN, Ferrari R, Rengo M, Paolantonio P, Vecchietti F, Laghi A (2009) Anatomic variations of the hepatic arteries in 250 patients studied with 64-row CT angiography. Eur Radiol, 19: 2765-2770. doi: 10.1007/s00330-009-1458-7.

7. Gadžijev E et al. (FULL LIST OF AUTHORS!!) (1996) Atlas of applied internal liver anatomy. Springer-Verlag, p. 75.

8. Gurgacz AM, Horbaczewska A, Klimek-Piotrowska W, Walocha J (2011) Variations in hepatic vascularisation: lack of a proper hepatic artery. Two case reports. Folia Morphol, 70: 130-134.

9. Hiatt J, Gabbay J, Busuttil RW (1994) Surgical anatomy of the hepatic arteries in 1000 cases. Ann Surg, 220: 50-52.

10. Jin GY (2008) Anatomical variations of the origin of the segment 4 hepatic artery and their clinical implications. Liver Transpl, 14: 1180-1184. doi: 10.1002/lt.21494.

11. Kamel IR, Kruskal JB, Pomfret EA, Keogan MT, Warmbrand G, Raptopoulos V (2001) Impact of multidetector CT on donor selection and surgical planning before liver adult right lobe liver transplantation. AJR, 176: 193-200.

12. Koops A, Wojciechowski B, Broering DC, Adam G, Krupski-Berdien G (2004) Anatomic variations of the hepatic arteries in 604 selective celiac and superior mesenteric angiographies. Surg Radiol Anat, 26: 239-244.

13. Kornasiewicz O, Krawczyk M, Paluszkiewicz R, Zieniewicz K, Hevelke P, Grzelak I, Pacho R, Rowiński O, Kaliciński P,
Kaminski A, Pawłowska J (2003) Anatomical alteration of the vascular tree observed during living related liver transplantation. Transplant Proc, 35: 2245-2247.

14. Michels N (1996) Newer anatomy of the liver and variant blood supply and collateral circulation. Am J Surg, 112: 337-347.

15. Onishi H, Kawarada Y, Das BC, Nakano K, Gadzijev EM, Ravnik D, Isaji S (2000). Surgical anatomy of the medial segment (S4) of the liver with special reference to bile ducts and vessels. Hepatogastroenterology, 47: 143-150.

16. Skórzewska A, Stajgis P, Grzymisławska M, Rojewska M, Krajecki M, Bruska M, Juszkat R (2014) Rare variations of hepatic arteries in association with variable origin of gastroduodenal artery found in multidetector computed tomography angiography. Folia Morphol, 73: 531-535. doi: 10.5603/FM.2014.0082.

17. Tzovaras G, Dervenis C (2006) Vascular injuries in laparoscopic cholecystectomy: an underestimated problem. Dig Surg, 23: 370-374.

18. Wang BG, Frober R (2009) Accessory extrahepatic arteries: Blood supply of a human liver by three arteries. A case report with brief literature review. Ann Anat, 191: 477-484. doi: 10.1016/j.aanat.2009.06.006.

19. Yamamoto S, Ericzon BG (2014) Domino liver transplantation as a valuable option. Transpl Int, 27: 27-28. doi: 10.1111/tri.12235. 